Western University

Scholarship@Western

Department of Economics Research Reports

Economics Working Papers Archive

1992

\title{
A Non-Cooperative View of Coalition Formation and the Core
}

Motty Perry

Philip Reny

Follow this and additional works at: https://ir.lib.uwo.ca/economicsresrpt

Part of the Economics Commons

Citation of this paper:

Perry, Motty, Philip Reny. "A Non-Cooperative View of Coalition Formation and the Core." Department of Economics Research Reports, 9203. London, ON: Department of Economics, University of Western Ontario (1992). 


\author{
RESEARCH REPORT 9203 \\ A NON-CODPERATIVE VIEW OF \\ COALITION FORMATION AND THE CORE \\ by \\ Motty Perry \\ and \\ Philip Reny
}

February 1992

Department of Economics

Social science Centre

University of Western Ontario

London. Ontario. Canada

$194=21992$

N6A $5 \mathrm{C2}$ 


\title{
A Non-Cooperative View of \\ Coalition Formation and the Core*
}

\author{
by \\ Motty Perry \\ and \\ Philip J. Reny
}

October 1991

*We wish to thank Murali Agastya, Kalyan Chatterjee, Ig Horstmann, and Arthur Robson for useful discussions. Reny acknowledges support from the Social Sciences and Humanities Research Council of Canada. 


\section{INTRODUCTION}

The notion of the core has attracted the interest of economists for more than 100 years. Much of the core's appeal stems from the intuitive and natural story behind it, the story that first motivated F.Y. Edgeworth in 1881. It runs as follows. An allocation which is not in the core is regarded as unstable. It is always the case, given such an allocation, that some individuals will be able to form a coalition and obtain an allocation that each strictly prefers.

Thus, the primary motivation for the core is non-cooperative in nature. Nonetheless, the core is not a non-cooperative solution concept. This is because, in particular, the possibilities for forming coalitions, and making offers and counteroffers, are not explicitly modelled.

In this work, we provide a non-cooperative implementation of the core. However, we do not merely implement the core. The nature of the game form employed is designed to reflect the motivating story as accurately as possible. The present results thus provide formal content to the usual intuitive justification for the core. In our view, the core would lose much of its appeal were it not possible to provide such a non-cooperative foundation.

The situations covered by our analysis have a payoff structure summarized by a transferable utility game in characteristic function form. This is embedded in a dynamic game in continuous time in which each player bargains over with whom to form a coalition and how to allocate the coalition's value among its members. The bargaining model employed allows flexibility in the negotiation process. In particular, there is no fixed timing or order of moves. At any positive time, any player can place a proposal on the table. A proposal consists of a coalition together with a suggested allocation of that coalition's value among its members. A coalition is formed if all its members accept the proposal before a new proposal is placed on the table. Once a new proposal is put forward, it automatically rescinds the previous one, so that only one proposal is on the table at any time. After a coalition, $\mathrm{S}$, is formed, any member can choose to leave and consume. In this event, all other members of $\mathrm{S}$ must also leave and 
consume their part of the agreed upon allocation. The remaining players, $\mathrm{NS}$, continue bargaining.

Continuous time is modelled along the lines of Perry and Reny (1991). A key feature of the model is that each player can time a proposal so that each member of the coalition to whom it is directed has an opportunity to react. Subgame perfectness and stationarity are the other main assumptions. Stationarity, in particular, may be too strong a restriction in some environments. Indeed, some environments may be less likely to yield core allocations than others.

In this setup we show that for $(v, N)$ a TU game:

(i) If an allocation is not in the core of $(v, N)$, then it cannot be supported as a stationary subgame perfect equilibrium (SSPE) outcome.

(ii) If $(v, N)$ is superadditive and totally balanced (in particular, if it is a market game) then:

(a) If payoffs are not discounted over time, then an allocation can be supported as an SSPE outcome if and only if it is in the core.

(b) If payoffs are discounted over time, then for every $\varepsilon>0$, an allocation can be supported as a stationary $\varepsilon$-subgame perfect equilibrium outcome if and only if it is in the $\varepsilon$-core.

The proof of (i) is constructed so that it reflects precisely the motivating story for the core. That is, assume, that some equilibrium allocation is not in the core. A blocking coalition is then able to deviate via a sequence of unilateral moves before the proposed equilibrium outcome is effected, guaranteeing each of its members more than they were to receive in the candidate equilibrium. This is a contradiction.

Hence, a necessary condition for the existence of an SSPE is that the underlying game, $(v, N)$, have a nonempty core. But, since subgame perfection is employed, an equilibrium must exist in every subgame; in particular, those in which only a subset of players remains. Thus a stronger condition is required for existence, namely that the game $(v, N)$ be totally balanced. 
When discounting is present, players willing to accept a proposal wish to do so as soon as possible. Given the continuous time setting, this is ill-defined. No subgame perfect equilibrium (stationary or otherwise) then exists. However, for every positive $\varepsilon$, a stationary $\varepsilon$-subgame perfect equilibrium does exist, and the set of such equilibrium outcomes is the $\varepsilon$-core.

There are by now a number of papers in which a non-cooperative game is designed to yield a cooperative solution. See, for instance, Harsanyi (1974), Selten (1981), Binmore (1985), Gul (1989), and Chatterjee et. al. (1990). Moldovanu and Winter (1991) reanalyze the game proposed by Selten (1981). Although the set of stationary subgame perfect equilibria in Selten's game depends on the assumed order of moves, they show that an allocation is in the core (of the underlying NTU game) if and only if for every possible order of moves it is an outcome of a stationary subgame perfect equilibrium.

The paper by Kalai, Postlewaite and Roberts (1979), henceforth KPR, is closest to ours in spirit. Theirs is a static game in which, simultaneously, each agent announces a coalition to which he wishes to belong and a feasible allocation for that coalition. Any coalition whose members' announcements match, obtains for its members the the announced allocation. Others receive their own value. KPR show that the set of strong Nash equilibria coincides with the core. The problem, of course, is that the requirement of strong Nash equilibrium begs the question.

The present work is also related to the literature on market games implementing the competitive outcome (see, for example, Gale (1987)). In such games traders meet in pairs each period according to some matching mechanism. The typical result is that for large economies, as the time between periods vanishes, every stationary equilibrium outcome converges to a competitive one (see, however, Rubinstein and Wolinsky (1985)). As we assume time to be continuous, it is enough in our game to replicate the economy sufficiently often in order to converge to a competitive outcome. This of course, is just a consequence of the core convergence theorem. 
In the next section we provide a heuristic description of the model, and show, by means of a few examples, the main features of the model, the roles of the assumptions, and the ideas behind the proofs. In section 3 a formal description of the model is given. Section 4 contains the theorems and their proofs.

\section{Overview of the Model and Examples}

Consider a situation in which the underlying opportunities can be summarized by a transferable utility game in characteristic function form, $G=(v, N)$, where $N=(1,2, \ldots, n)$ is the (finite) set of players and $v: 2^{N} \rightarrow R$ associates with every subset, $S$, of $N$ (or equivalently, every coalition) its value $\mathbf{v}(\mathbf{S})$.

The following extensive form describes how coalitions form and associated allocations are achieved. Time is continuous. The game starts at $\mathrm{t}=0$ at which time a player can choose to make a proposal or to be quiet. At every positive time t, a player can choose to make a proposal, accept a current proposal, be quiet, or leave and consume. We now elaborate on these possibilities.

A proposal $(x, S)$ by player $i \in N$, consists of a coalition $S$ (where i need not be a member of $S)$, and an allocation $\left(x_{j}\right)_{j \in S}$, the sum of whose components does not exceed $v(S)$. If player i makes a proposal $(x, S)$ at time $t$, then this proposal is effective so long as no new proposal is made. Thus, only one proposal is dealt with at a time, and once a new proposal is made the previous one is no longer in effect.

A proposal becomes binding when it is accepted by all members of the relevant coalition while it is effective. Once a player accepts a proposal, he must remain quiet until that proposal becomes binding, or until it is replaced by a new proposal. (This restriction serves a purely technical role and is discussed at length in example $1 \mathrm{~b}$ below.)

Once a proposal, $(\mathrm{x}, \mathrm{S})$, becomes binding, we say that the coalition $S$ has formed and has accepted $(\mathrm{x}, \mathrm{S})$. Any player, $\mathrm{i}$, in $\mathrm{S}$ can then choose to leave and consume. He thereby obtains the payoff $x_{j}$, according to the binding proposal $(x, S)$. When this occurs, every $j \in$ 
$S \backslash\{i\}$ must also leave and consume, thereby obtaining a payoff of $x_{j}$. Thus, each member, $i$, of $S$ can ensure himself a payoff of $x_{i}$ once $(x, S)$ becomes binding. So long as no member of $S$ leaves, each can make and accept proposals.

At any time during the course of play then, there may be a number of binding proposals $\left(y^{1}, s^{1}\right),\left(y^{2}, s^{2}\right), \ldots,\left(y^{m}, s^{m}\right)$, say, among players who have not left. In order to submit a proposal $(\mathrm{x}, \mathrm{S})$ in the presence of such binding proposals, it is assumed that if $i \in S \cap S^{j}$, then $S S_{2} S^{j}$. Thus, players are not allowed to a make proposal to a strict subset of coalitions that have already formed. This restriction is for simplicity only. Finally, if player i never leaves to consume, his payoff is $d_{i} \leq v(i)$.

It is assumed that for all times $t$, and after any history up to time $t$, players are not allowed to choose to make a proposal, accept a proposal, or leave to consume either "just before" or "just after" time $t$. That is, for any time $t$, for every history up to $t$ and every vector of actions available to the players at time $t$ according to the history, there is an $\varepsilon>0$, such that each player is quiet, according to his strategy, in the open intervals $(t-\varepsilon, t)$ and $(t, t+\varepsilon)$. As is well known, without some such assumption, the continuous-time game is not well-defined. This particular assumption, however, is somewhat more than is needed for that purpose. Indeed, it provides not only the technical service of ensuring well-definedness, it plays a central role in obtaining the results.

The solution concept applied to this game is stationary subgame perfect equilibrium (SSPE). That is, stationary strategies constituting a subgame perfect equilibrium (among all strategies, not only stationary ones). A strategy is stationary if, after any history, it depends only upon the set of players remaining, the current proposal, the set of players who have accepted it, and the set of binding proposals. ${ }^{1}$ Before the formal details of the game are

1 We also allow stationary strategies to depend, in a limited sense, upon time. Indeed, some time dependence is necessary. This is discussed fully below. For the intuition, however, it is best at this point to put this (largely technical) issue aside. 
described and the proofs of the theorems presented, it may be helpful to consider a few examples which illustrate the main ideas.

\section{Example 1:}

There are two buyers and one seller. The seller (player 1) owns one unit of a divisible good that is of no value to him. The two buyers (players 2 and 3 ) each value the good at one dollar. A TU game describing this situation is given by:

$$
N=\{1,2,3\}, v(i)=v(2,3)=0 \text { for } i \in N \text {, and } v(1,2)=v(1,3)=v(N)=1 \text {. }
$$

In this game the core consists of a single point, namely $x^{*}=(1,0,0)$. This corresponds to the competitive outcome as excess demand results in the seller obtaining the entire surplus. We now show (informally) that only the division $x^{*}$ can be supported as a stationary subgame perfect equilibrium outcome.

Assume, by way of contradiction, that $\left(\mathrm{x}_{1}, \mathrm{x}_{2}, \mathrm{x}_{3}\right)$ is a stationary subgame perfect equilibrium outcome with $x_{1}<1$, and assume without loss that $x_{2} \leq x_{3}$. Let $\hat{t}>0$ be such that for all $\mathrm{t}<\hat{\mathrm{t}}$ no player accepts any proposal according to the strategies. By assumption, such a time must exist. ${ }^{2}$ Consider the following deviation by player 1 : At time $t_{1}=\hat{t} / 2$ propose $\left(x_{1}+\left(1-x_{1}-x_{2}\right) / 2, x_{2}+\left(1-x_{1}-x_{2}\right) / 2,\{1,2\}\right)$ and at time $t_{2}>t_{1}$ accept this proposal, where $t_{2}$ is such that all players, according to their strategies, are quiet in the interval $\left(t_{1}, t_{2}\right]$. Again, by assumption, such a time $t_{2}$ is guaranteed to exist. Consider the continuation after $t_{2}$. Let $t_{3}$ be the first time after $t_{2}$ at which some player is not quiet according to his strategy. Player 2 can guarantee himself a payoff of $x_{2}+\left(1-x_{1}-x_{2}\right) / 2$ by accepting the above proposal at any time $t \in\left(t_{2}, t_{3}\right)$ If, according to his strategy, he is to accept this proposal, then the deviation is

2Indeed, given the empty history up to time zero, and the actions of the players at time zero as specified by their strategies, there is an $\varepsilon>0$ such that all players are quiet in the interval $(0, \varepsilon)$. Since no player can accept a proposal at time zero, putting $\hat{t}=\varepsilon$ will suffice. 
successful. But this contradicts $\mathrm{x}$ being an equilibrium. So, it must be that player 2 , according to his strategy, does not accept 1's proposal. It follows that, according to the equilibrium strategies, at time $t_{3}$ someone makes a proposal, say $(y, T)$, and player 2 subsequently obtains a payoff of at least $x_{2}+\left(1-x_{1}-x_{2}\right) / 2$. Note then that by stationarity, whenever (a) all players remain, (b) the current proposal is $(y, T),(c)$ no one has accepted it, and (d) there are no binding proposals, the subsequent outcome, according to the equilibrium strategies, gives player 2 at least $x_{2}+\left(1-x_{1}-x_{2}\right) / 2$. But this contradicts $\left(x_{1}, x_{2}, x_{3}\right)$ being the equilibrium outcome. For player 2 can deviate at any time $t<\hat{t}$ by proposing $(y, T)$, thereby rendering (a)-(d) all true and ensuring himself (by stationarity) a payoff exceeding $x_{2}$.

The above argument shows that the only candidate for an equilibrium outcome is $x^{*}=(1,0,0)$. We now present (again, informally) a profile of stationary subgame perfect equilibrium strategies yielding the outcome $x^{*}$.

If the current time is not an integer, then all players are quiet. Suppose now that the current time is an integer, and player 1 does not belong to any coalition that has formed. If the current proposal is $\left(x^{*}, N\right)$, then each player who has not yet accepted it, does so. If all players have accepted it, then all leave. If the current proposal is not $\left(x^{*}, N\right)$ and player 1 has not accepted it, then player 1 proposes $\left(x^{*}, N\right)$ and the others are quiet. If player 1 has accepted it, then the others accept it as well.

It remains to describe players' strategies after histories in which player 1 belongs to a coalition that has formed. So, suppose that a coalition, $S$, containing player 1 , has formed and has accepted $(y, S)$. Let $d=1-\sum_{i \in S} y_{i}$ be the amount of surplus "wasted" by the binding proposal $(y, S)$. To obtain each players' action, consult once more the previous paragraph, while ignoring the second half of the second sentence, and replace $x *$ throughout with $\left(y_{1}+d, y_{2}, y_{3}\right)$. It is easy to see that the above strategies constitute a subgame perfect equilibrium, are stationary, and support the outcome $x^{*}$.

The one seller/two buyers example above is often used to discredit the concept of the core. It is, the argument goes, inconceivable that the two buyers will end up with no surplus. 
They can, for example, tacitly bargain with the seller as a coalition, and this might yield both a strictly positive payoff. Note, however, that for any such payoffs the buyers might secure, there is an offer that the seller both prefers and can make to one of them which, from that one's point of view, is strictly better. Stationarity guarantees that this offer must be accepted. This exemplifies the general role stationarity plays in obtaining the result that only core points can be supported as equilibrium outcomes.

The example above also gives the essentials of the general argument establishing that only core allocations can be supported in equilibrium. It follows that a necessary condition for the existence of an SSPE is that the core of the TU game be non empty. But this is not sufficient. Given subgame perfection, histories after which any subset of the players remain must also be considered. A necessary condition for the existence of an SSPE in such a subgame is that the TU game restricted to this subset of players also have a nonempty core. It thus follows that a necessary condition for the existence of an SSPE is that the game $(v, N)$ be totally balanced.

Is total balancedness a sufficient condition for existence? What about histories after which some binding proposals are present and all players remain, say? After such a history the underlying game, $(\mathrm{v}, \mathrm{N})$, is no longer relevant since some player, i say, may be able to guarantee himself more than $\mathbf{v}(\mathrm{i})$ by leaving to consume his share of a binding proposal. Indeed, the now relevant TU game is identical to $(v, N)$ except that each member of a formed coalition can guarantee himself a payoff equal to his share of the corresponding binding proposal. Now, in order for an SSPE to exist, any candidate continuation equilibrium allocation, $x$, must be immune to any feasible improvement. Since strict subsets of formed coalitions cannot form, this amounts to $\mathrm{x}$ being unblockable by any coalition not a strict subset of a formed coalition. When is such an $x$ guaranteed to exist? It turns out that for TU games, the assumption of total balancedness is enough to ensure the existence of such an $\mathbf{x}$, regardless 
of the history. ${ }^{3}$ The next example serves to illustrate the importance of keeping track, given a history, of the relevant TU game, and how an appropriate continuation equilibrium outcome is constructed.

\section{Example 1a:}

Consider the following attempt at constructing an SSPE for the one seller/two buyers game of example 1. For every subset of players, $S$, let $x(S)$ be an element of the core of the game $(v, N)$ restricted to $S$, and put $x(N)=x^{*}$. This is possible since $(v, N)$ is totally balanced. For any history, if the set of remaining players, according to that history, is $S$, then construct the continuation strategies so that the subsequent outcome is $x(S)$, a point in the core of the game $(v, N)$ restricted to $S$.

The above procedure does not produce an SSPE as we now show. Take a history according to which all players remain, and $((.4, .4),\{1,2\})$ is the only binding proposal. According to the procedure outlined above the subsequent outcome will be $x(N)=x^{*}=(1,0,0)$. But this gives player 2 a continuation payoff of zero, while player 2 , by leaving, can obtain a payoff of .4. Hence, any equilibrium continuation must give player 2 a payoff of at least .4 , the amount he can unilaterally guarantee himself by virtue of the existing binding proposal.

As previously described, the correct procedure for constructing an equilibrium continuation involves strategies that permit the players to agree on an outcome that is unblockable, in the context of the relevant TU game, by coalitions that can feasibly form. It is not difficult to see that, after the history given above, any outcome $\left(x_{1}, x_{2}, x_{3}\right)$ with $x_{1}+x_{2}=1$ and $x_{1}, x_{2} \geq .4$ will suffice.

In general, there may be many binding proposals present after some history, and the continuation outcome may require the joining of two or more previously formed coalitions. Of

${ }^{3}$ This result fails in the NTU case. 
course, if the game is superadditive there is never any loss in joining previously formed coalitions. On occasion, however, some must be joined. The proof of proposition 2 contains the general construction of an appropriate equilibrium continuation outcome, after any history.

The assumption that, upon accepting a proposal, a player must be quiet until either the proposal is replaced by another or until it becomes binding, is somewhat restrictive and so merits some discussion. The example to follow shows that this assumption is needed to ensure the existence of an SSPE. Moreover, it indicates that this assumption plays only a technical, rather than a more substantive role in the analysis.

\section{Example 1b:}

Consider again the one seller/two buyers game of example 1 and, contrary to our assumption, allow players, upon accepting a proposal, to make a new proposal if they wish. It is easy to see that this additional flexibility does not affect at all the argument that points other than $x^{*}=(1,0,0)$ cannot be supported as an SSPE allocation. Thus, as before, $x^{*}$ is the only candidate for an equilibrium allocation. Consider, however, a history according to which, (a) all players remain, (b) there are no binding proposals, (c) the current proposal is $x=(0, .5, .5)$ to the grand coalition, and (d) players 1 and 2 have accepted it. Because $x^{*}$ is the only candidate for an equilibrium outcome, it follows (by stationarity) that if player 1 now proposes $x^{*}$ to the grand coalition, his payoff will be 1 and players 2 and 3 will obtain payoffs of zero. However, if player 3 accepts the current proposal, rendering it binding, his payoff will be .5 in the continuation, and player 1's will be zero. Hence, player 1 wishes to replace the current proposal before player 3 accepts it, and player 3 wishes to accept it before player 1 replaces it. With time continuous, there can be no equilibrium in this race to be the first to move after a given time.

To recap, the assumption under discussion is not needed to guarantee that non core points cannot be supported as equilibrium outcomes. It plays a role only in ensuring that an SSPE supporting a point in the core can be constructed and then only to ensure that irrational 
(out-of-equilibrium) proposals do not produce histories having no equilibrium continuation.

In the present model, as distinct from most of its predecessors, coalitions are not forced to leave and consume once they form (Gul (1989) is an exception). Whether to stay and continue negotiating or to leave and consume is endogenously determined by the players' strategies. While this seems to be a natural assumption, it is also necessary, in the present continuous-time context, to ensure the existence of an equilibrium. The final example is designed to illustrate this.

\section{Example 2:}

Add both an additional buyer and seller to example 1 . Let players 1 and 2 be the sellers and 3,4,5 the buyers. Again, each seller has a single divisible good of no value to him, and each buyer values each good alone at one dollar and both goods together at one dollar as well. The unique core point is $\mathrm{x}^{*}=(1,1,0,0,0)$.

Consider now a history according to which there are no binding proposals and the current proposal involves the allocation $x=(0,1 / 3,1 / 3,1 / 3)$ to the coalition $(1,3,4,5\}$. Suppose also that, according to this history, players 1,3 and 4 , have accepted this proposal. Now, if player 5 accepts this proposal, rendering it binding, his payoff in the continuation is at least $1 / 3$. If, however, this proposal is replaced by another before the coalition $\{1,3,4,5\}$ forms, then in the continuation, the resulting division must (by stationarity) be $(1,1,0,0,0)$, the unique core point. It follows that player 5 must accept the proposal and that the coalition $\{1,3,4,5\}$ must form. However, if when the coalition $\{1,3,4,5\}$ forms, each member, contrary to our assumption, must leave and consume, player 2 will obtain a payoff of zero. But this cannot be an equilibrium, since player 2 can, before player 5 accepts the current proposal, replace it by another and thereby obtain a payoff of 1 . So, player 2 wishes to replace the proposal before player 5 accepts it, and player 5 wishes to accept it before player 2 replaces it. Hence, under the assumption that coalitions, once formed, must leave, there is no equilibrium in this game.

On the other hand, if, as we assume, coalitions are not forced to leave, then one can 
construct the following continuation. At the next integer time, $\overline{\mathrm{t}}$, player 5 accepts the proposal and the coalition $\{1,3,4,5\}$ forms, but does not leave. At time $\bar{t}+1$, player 2 proposes the allocation $(1,0,1 / 3,1 / 3,1 / 3)$ to the grand coalition. At time $\overline{\mathrm{t}}+2$ all players accept this proposal, and at time $\overline{\mathrm{t}}+3$ all leave and consume.

\section{The Model}

Histories: Let q stand for the choice to be quiet, $\ell$ for the choice to leave, and a for the choice to accept the current proposal. Let

$$
P=\left\{(x, S) \mid S \subseteq N, x_{i} \geq v(i) \text { for all } i \in S, \sum_{i \in S} x_{i} \leq v(S)\right\}
$$

denote the set of possible proposals.

A history, $h$, up to time $t$ is an $n$-tuple of functions: $h=\left(h_{1}, h_{2}, \ldots . . h_{n}\right)$, where for each $i=1,2, \ldots, n, h_{i}:[0, t) \longrightarrow P \cup\{q, a, \ell\}, h_{i}^{-1}(P \cup\{a\})$ is finite, and $h_{i}^{-1}(\{\ell\})$ is either zero or one.

Denote by:

(i) $\mathrm{H}$, the set of all histories,

(ii) $H(t)$, the set of all histories up to time $t$,

(iii) $p(h)$, the current proposal according to $h$,

(iv) $\tau(h)$ for $h \in H(t)$, the amount of time, according to $h$, that has elapsed up to $t$ since $\mathrm{p}(\mathrm{h})$ was proposed,

(v) $A(h)$, the set of players who have accepted $p(h)$ according to $h$,

(vi) $\quad \mathrm{N}(\mathrm{h})$, the set of players who have not yet left to consume according to $h$, and

(vii) $\Pi(h)$, the set of current binding proposals among players in $N(h)$ according to $h$.

With the exception of $p(h), \tau(h)$ and $A(h)$, all entries in the above list are well-defined owing to the fact that but for finitely many points in time, all players are quiet according to any history. To render $p(h)$ well-defined, it suffices to define $p(h) \equiv \phi$ if, according to $h$, any one of the following holds: the most recent proposal made coincides with the simultaneous announcement of a distinct proposal; no proposal has been made; or there is a most recent 
proposal, $(x, S)$, and it has been accepted by $S$. The remaining two entries, $\tau(h)$ and $A(h)$, are now taken care of by setting $\tau(\mathrm{h})=0$ and $\mathrm{A}(\mathrm{h})=\phi$, whenever $\mathrm{p}(\mathrm{h})=\phi$.

Payoffs: If a coalition, $S$, accepts $(x, S)$ and subsequently leaves the game, then each $i \in S$ receives the payoff $x_{i}$. If player $i$ never leaves, he obtains a payoff of $d_{i} \leq v(i)$.

Strategies: A strategy, $\mathrm{f}_{\mathrm{i}}$, for player $\mathrm{i}$ specifies, for each history, a choice for player $\mathrm{i}$. That is: $f_{i}: H \rightarrow P \cup\{q, a, \ell\}$ for all $i \in N$.

We say that player i has accepted the current proposal $(x, S)$ according to $h \in H(t)$ if the current proposal was made at time $\bar{t}<t$ and $h_{i}\left(t^{\prime}\right)=a$ for some $t^{\prime} \in(\bar{t}, t)$. Also, denote by $h \mid t$ the restriction of $h \in H$ to $[0, t)$.

We impose the following restrictions upon strategies:

(S1) If, according to $h \in H(t)$, $i$ has accepted the current offer, then $f_{i}(h)=q$.

(S2) If, according to $h \in H(t),(x, S)$ is a current binding proposal and $f_{i}(h)=\left(y, S^{\prime}\right)$, then $S \cap S^{\prime} \neq \emptyset$ implies that $S \subseteq S^{\prime}$. If, according to $h \in H(t), i$ is not a member of any formed coalition, then $\mathrm{f}_{\mathrm{i}}(\mathrm{h}) \neq l$

$\forall \mathrm{i}, \forall \overline{\mathrm{t}}>0, \forall \mathrm{h} \in \mathrm{H}(\overline{\mathrm{t}}), \forall \mathrm{t} \in[0, \overline{\mathrm{t}}), \exists \varepsilon>0$ s.t $\mathrm{f}_{\mathrm{i}}(\mathrm{h} \mid \tau)=\mathrm{q} \forall \tau \geq 0$ satisfying $\tau \in(t-\varepsilon, t+\varepsilon) \backslash\{t\}$.

Conditions (S1)-(S3) merely ensure that the choices made by the players, according to their strategies, are consistent with the rules of the game as previously described. For instance, (S1) ensures that a player is quiet after accepting a proposal until it is either accepted by the associated coalition or replaced by a new proposal. Condition (S2) guarantees that proposals made to any member of a formed coalition must include every member of that coalition, while (S3) ensures that no player can leave without having first accepted a proposal. In particular, in order to leave and consume his own value, any player, $i$, must first propose ( $v(i),\{i\})$, accept it, and then leave. In the present continuous-time context this does not restrict in any way a 
player's freedom to leave. It does, however, ensure that the other players are given ample warning before anyone leaves.

Condition (S4) plays a dual role. Its more substantive one is to ensure that no player wishes to make a proposal, accept a proposal, or leave, as near as possible, yet before or after, any fixed time $t \geq 0$. This guarantees, for instance, that if player 1 , say, recognizes that according to player 2's strategy, player 2 intends to leave, then player 1 can make a proposal to player 2, with a view to convincing him to stay, at a time during which all others are quiet and player $\mathbf{2}$ has not yet left. This is clearly important in order to obtain only core outcomes. For it may be that players 1 and 2 must form a coalition to attain a core allocation. This could not be guaranteed were player 1 not ensured of the opportunity to "convince" player 2 to stay by, say, making an appropriate blocking proposal.

As a by-product, condition (S4) also serves a purely technical role. It guarantees that the game is well-defined. To be more precise requires a short discussion. Call $h=\left(h_{1}, \ldots, h_{n}\right) a$ path, if for some $\bar{t} \in(0, \infty]$, (a) $h \mid t \in H(t)$ for every $t \in[0, \bar{t})$ and (b) $\bar{t}$ is finite iff it is the first time (hence the only time) such that all players have left according to $h$ (i.e. the smallest $t^{\prime}$ with $h_{i}\left(t^{\prime}\right)=\ell$ for all $\left.i \in M N\left(h \mid t^{\prime}\right)\right)$. It is straightforward to determine from every path a unique payoff vector for the players according to the rules described above. Thus, to demonstrate that the game is well-defined, it suffices to show that any strategy-tuple, $f$, satisfying (S1)-(S4) always induces a unique continuation path. That is, given any $h^{\prime} \in H\left(t^{\prime}\right)$ it suffices to show that there is a unique path, $h$, with its associated $\bar{t} \in(0, \infty]$ satisfying $(i) h=$ $h^{\prime}$ on the domain of $h^{\prime}$, and (ii) $f(h \mid t)=h(t)$ for every $t \in\left[t^{\prime}, \bar{t}\right]$. That (S4) is (more than) enough to guarantee this is by now rather well-known (see, for instance, Bergin and McLeod (1989), Stinchcombe (1988), and Perry and Reny (1991)). Consequently, (S4) ensures that after any history, any strategy-tuple induces a unique continuation-path which, by definition, takes on the value q ("quiet") for every player for all but finitely many points in any bounded interval of time. In what follows, let $u_{i}(f \mid h)$ denote player i's payoff determined from the 
continuation-path induced by the strategy-tuple $f$ after $h \in H$, and let $F_{i}$ denote the set of strategies for i satisfying (S1) - (S4).

\section{Solution concept:}

A strategy profile $\hat{\mathrm{f}}=\left(\hat{\mathrm{f}}_{1}, \hat{\mathrm{f}}_{2}, \ldots \hat{\mathrm{f}}_{\mathrm{n}}\right)$ is a stationary subgame perfect equilibrium (SSPE) if:

(E1) Perfection: For all $i \in \mathrm{N}$ and for all $h \in \mathrm{H}$,

$$
u_{i}\left(\left(\hat{f}_{i}, \hat{f}_{-i}\right) \mid h\right) \geq u_{i}\left(\left(f_{i}, \hat{f}_{-i}\right) \mid h\right) \text { for all } f_{i} \in F_{i} \text {, }
$$

(E2) Stationarity: Players' strategies depend only upon the time that has elapsed since the current proposal was submitted, the current proposal, the set of players who have accepted it, the set of players remaining in the game, and the set of binding proposals among players still in the game. That is, if $h, h^{\prime} \in H$ satisfy $\tau(h)=\tau\left(h^{\prime}\right), p(h)=p\left(h^{\prime}\right)$, $A(h)=A\left(h^{\prime}\right), N(h)=N\left(h^{\prime}\right)$, and $\Pi(h)=\Pi\left(h^{\prime}\right)$, then $\hat{f}_{i}(h)=\hat{f}\left(h^{\prime}\right)$.

For any history, $h$, call $(\tau(h), p(h), A(h), N(h), \Pi(h))$ the state according to $h$. Note that the inclusion of $\tau$ in the state variable has the effect of allowing stationary strategies to depend, in a limited sense, upon time. Some time dependence is necessary to ensure existence in the present environment. For instance, in the one seller/two buyers example, in order to achieve the unique core point, $\mathrm{x}^{*}$, as an SSPE outcome, a proposal implying the allocation $\mathrm{x}^{*}$ must be made and then subsequently accepted. However, if a player's strategy instructs him to accept this proposal with no reference to time, then it must be accepted "as soon as possible", yet after, it is made. This violates (S4). To avoid this difficulty, the proposal can instead be accepted, say, one unit of time after it is made. This is compatible with condition (E2).

\section{The Theorems}

Theorem 1: If the outcome $x$ can be supported as a stationary subgame perfect equilibrium, then $x$ is in the core of $(v, N)$. 
Proof: Suppose that $\mathrm{x}$ is not in the core. We shall show by way of contradiction that $\mathrm{x}$ cannot be supported by an SSPE. So suppose that $x$ can be supported by the SSPE strategy-tuple $f=\left(f_{1}, f_{2}, \ldots f_{n}\right)$. Clearly $x_{i} \geq v(i)$ for every $i \in N$. Further, since $x$ is not in the core, there is a proposal, $(y, S)$, with $y_{i}>x_{i}$ for all $i \in S$. Let $S=\{1,2, \ldots, k\}$.

Suppose that there is a time, $t$, and a history, $h \in H(t)$, such that (i) $N(h)=N$, (ii) $\Pi(h)=\phi$ (iii) players $1,2, \ldots, k-1$ have accepted $(y, S)$ according to $h$, and (iv) $f_{i}(h)=q$ for all $i=1,2, \ldots . n$.

Claim: Player $\mathbf{k}$ will, according to his strategy and before any proposal is subsequently made, accept $(\mathbf{y}, \mathbf{S})$.

Proof of Claim: If a proposal, $(z, T)$, is made before player $k$ accepts $(y, S)$, then at the time it is made, the state variable will be rendered equal to $(0,(\mathrm{z}, \mathrm{T}), \phi, \mathrm{N}, \phi)$. Moreover, because player $k$ could have accepted the previous proposal, guaranteeing himself a payoff of $y_{k}>x_{k}$ ' but chose instead to allow it to be replaced by $(z, T)$, the continuation must yield $k$ a payoff of say, $w_{k} \geq y_{k}$. Therefore, by stationarity, whenever the state variable takes on the value $(0,(z, T), \phi, N, \phi)$, player k's subsequent payoff according to $f$ is $w_{k} \geq y_{k}>x_{k}$. But this contradicts $f$ being an equilibrium since $k$ can render the state equal to $(0,(z, T), \phi, N, \phi)$ by proposing $(z, T)$ near enough time zero.

Next, suppose that there is a time $t$ and a history $h \in H(t)$ such that (i) $N(h)=h$, (ii) every player $i=1,2, \ldots k-2$ has accepted $(y, S)$ according to $h$, and (iii) $f_{i}(h)=q$ for $i=1,2, \ldots n$.

Claim: The proposal $(y, S)$ will be accepted by players $k-1$ and $k$ (in some order) before any new proposal is made.

Proof of Claim: Note that player $k-1$ can guarantee himself a payoff of $y_{k-1}>x_{k-1}$ by accepting $(y, S)$ at time $t$. This is because for some amount of time after $k-1$ 's acceptance, by (S4) all player must be quiet. But then at any time during this interval, the history will be such that player $k$ will accept $(y, S)$, by the previous claim. Thus, indeed player $k-1$ can guarantee himself a payoff of $y_{k-1}$. Now then, in the continuation after $h$, either no new proposal is made according to $f$, or one is. In the former case, $k-1$ and $k$ would receive 
payoffs of $d_{k-1}$ and $d_{k}$ respectively, contradicting k-1's ability to achieve a payoff of at least $y_{k-1}>x_{k-1} \geq d_{k-1}$. In the latter case we arrive at a contradiction as in the previous claim. Player $k-1$ 's payoff subsequent to the new proposal must be at least $y_{k-1}$. But $k-1$ could then himself make this proposal near enough time zero rendering the state variable such that his subsequent payoff (by stationarity) would be at least $y_{k-1}>x_{k-1}$.

Proceeding inductively in this manner establishes that for any time $t$ and any history $h \in H(t)$ such that (i) $N(h)=N$, (ii) player 1 has accepted $(y, S)$ according to $h$, and (iii) $f_{i}(h)=q$ for $i=1,2, \ldots, n$, the continuation according to $\left(f_{1}, f_{2}, \ldots, f_{n}\right)$ involves the solution, $S$, accepting $(y, S)$.

But this contradicts $x$ being an SSPE since player 1 can always offer $(y, S)$ and then accept it sufficiently near time zero so that such a history is generated, thereby ensuring himself a payoff of $y_{1}>x_{1}$.

Q.E.D.

Remark 1: Note how the line of proof employed above follows precisely the "story" that is usually used to motivate the notion of the core. An outcome which is not in the core cannot be supported since a blocking coalition is able to coordinate a mutually preferred proposal before the candidate outcomes's proposal is accepted.

Remark 2: The ability to announce a proposal and have sufficient time for all members of the associated coalition to sequentially accept it before the candidate outcome is consumed is an essential property. One that requires continuous time. Thus, continuous time is an essential feature of the model.

Remark 3: An implication of Theorem 1 is that $(v, N)$ having a nonempty core is necessary for the existence of an SSPE. Indeed, since the arguments above apply after any history in which only a subset of the players remain, the game $(v, N)$ restricted to any subset of players must also have a nonempty core. Consequently, if an SSPE is to exist, $(v, N)$ must be totally balanced. We now establish that for superadditive TU games this condition is, in fact, sufficient. 
Theorem 2: If $(v, N)$ is superadditive and totally balanced, and $x$ is in the core of $(v, N)$, then $x$ can be supported as an SSPE outcome.

Before providing a proof, we remind the reader of the feature highlighted by example 1a. Namely, the need to keep track of the relevant TU game. When there are binding proposals present, each player can guarantee himself the payoff associated with his part of the binding proposal. Moreover as shown in example 2, some players may be needed in order to achieve a core allocation and so must be convinced to stay despite having reached a previous agreement. The function $\mathrm{z}(\cdot, \cdot)$ defined below is designed to provide allocations that guarantee each member of a formed coalition at least as much as his binding agreement ensures him (see part (a) of Lemma 1 in the appendix). Moreover, no feasible coalition can block the allocation given by $z(\cdot, \cdot)$ (this follows from part (b) of Lemma 1 ). The strategies to be constructed below will employ $z(\cdot, \cdot)$ to determine the continuation outcome subsequent to any history. In particular, those according to which binding proposals are present.

Proof: We provide SSPE strategies supporting the outcome $x$. For each nonempty ScN let $(v, S)$ denote the TU game with player set $S$ and characteristic function $v$ restricted to $S$. Choose for each such $S \neq N$ an element $x(S)$ from the core of $(v, S)$, and set $x(N)=x$. This is possible because $(v, N)$ is totally balanced.

Given $\Pi=\left\{\left(y^{1}, s^{1}\right),\left(y^{2}, s^{2}\right), \ldots,\left(y^{m}, s^{m}\right)\right\}(m=0$ means that $\Pi=\phi)$ and $S \subseteq N$ such that for each $k$, either $S^{k} \subseteq S$ or $S^{k} \cap S=\phi$, define for every $i \in S, z_{i}(\Pi, S)$ as follows

$$
z_{i}(\Pi, S)= \begin{cases}y_{i}^{k}+\frac{\sum_{j \in S^{k}}\left(x_{j}(S)-y_{j}^{k}\right)}{\left|S^{k}\right|}, & \text { if } i \in S^{k} \\ x_{i}(S) & \text { if } i \in S \backslash{\underset{k=1}{\cup} S^{k}}^{m}\end{cases}
$$


For $h \in H$, if $\Pi(h)=\Pi$ above, and $p(h)=(y, S)$ then permuting the indices on the elements of $\Pi(h)$ if necessary, there is an $r \leq m$ such that $S_{2} S^{1} \cup S^{2} \ldots \cup S^{r}(r=0$ means that the union is empty). Define $\hat{\Pi}(h)$ by $\hat{\Pi}(h)=\left\{(y, S),\left(y^{r+1}, S^{r+1}\right), \ldots,\left(y^{m}, S^{m}\right)\right\}$. Note that if $(y, S)$ were accepted by the members of $S, \Pi$ would change to $\hat{\Pi}$. To simplify notation, $z_{i}(\Pi(h), N(h))$ and $z_{i}(\hat{\Pi}(h), N(h))$ will be denoted by $z_{i}(h)$ and $\hat{z}_{i}(h)$, respectively. Note that $\hat{z}_{i}$ depends only upon $p(h), \Pi(h)$ and $N(h)$.

We now provide the desired strategies. For every $t>0, h \in H(t)$ and $i \in N$,

(i) if $\boldsymbol{t}(\mathrm{h})$ is not a positive integer, then $f_{i}(h)=q$.

(ii) if $\tau(\mathrm{h})$ is a positive integer

(a) if $p(h)=(y, S)$, and $\hat{z}_{i}(h) \geq z_{i}(h)$ for all $i \in S \backslash A(h)$, then

$$
f_{i}(h)= \begin{cases}a, & \text { if } i \in S \backslash A(h) \\ q, & \text { otherwise }\end{cases}
$$

(b) Otherwise ${ }^{4}$

$$
f_{i}(h)=\left\{\begin{array}{l}
\ell, \quad \text { if } i \in N(h) \backslash A(h) \text { and } \Pi(h)=\{(z(h), N(h))\} \\
(z(h), N(h)) \text { if } i \in N(h) \backslash A(h) \text { and } \Pi(h) \neq\{(z(h), N(h))\} \\
q, \quad \text { ot herwise. }
\end{array}\right.
$$

These strategies are certainly stationary since they depend only upon $\tau(h), p(h), A(h), N(h)$ and $\Pi(h)$. In addition, the path generated by $f=\left(f_{1}, \ldots f_{n}\right)$ has all players propose $(x, N)$ at time 1 , accept $(x, N)$ at time 2 , and leave at time 3. At all other times the players are quiet according to this path. Therefore $f$ yields the outcome $x$. (For a more formal treatment, see Lemma 2 and put $h=\phi$.) It remains to check that $f$ constitutes a subgame perfect equilibrium.

Note that if $f_{j}^{l}(h)=\ell$ for some $i \in N(h) \backslash(j)$ then according to $f_{i}$ it must be the case that $\Pi(h)=\{(z(h), N(h))\}$. Hence, if player $i$ leaves, every player in $N(h)$ chooses to leave as well

4For $h$ with $f_{i}(h)=(z(h), N(h))$ as specified on occasion in (b), we must ensure that $(\mathrm{z}(\mathrm{h}), \mathrm{N}(\mathrm{h}))$ is a feasible proposal. This is the only place where superadditivity is used. 
and so $f_{j}$ is clearly optimal given such a history. So, given $t>0$, consider $h \in H(t)$ such that $j \notin A(h)$ and $f_{i}(h) \notin \ell$ for all $i \in N(h) \backslash\{j\}$. It suffices to show that, given such a history, $f_{j}$ is best for $j$ if the others employ $f_{i}$.

Let $f_{j}^{\prime}$ denote another strategy for $j$. Together, $f_{j}^{\prime}$ and $f_{-j}$ generate a unique path $h^{\prime}$. Since (by part (a) of Lemma 1) $z_{j}(h) \geq v(j), j$ does no better if, according to $h^{\prime}, j$ never leaves. So suppose that, according to $h^{\prime}, j$ leaves at time $t^{\prime}$ with the proposal $\left(y^{\prime}, S^{\prime}\right)$, and so receives a payoff of $y_{j}^{\prime}$. Since $\left(y^{\prime}, S^{\prime}\right)$ must have been accepted by each member of $S^{\prime}$ before time $t^{\prime}$, we have $\left(y^{\prime}, S^{\prime}\right) \in \Pi\left(h^{\prime} \mid t^{\prime}\right)$ so that by Lemma $1 z_{j}\left(h^{\prime} \mid t^{\prime}\right) \geq y_{j}^{\prime}$. Without loss of generality then, suppose that $\mathrm{f}_{\mathrm{j}}^{\prime}$ yields $\mathrm{j}$ a payoff of $\mathrm{z}_{\mathrm{j}}\left(\mathrm{h}^{\prime} \mid \mathrm{t}^{\prime}\right)$.

We consider three exhaustive, although not mutually exclusive, cases:

Case A: $\quad A(h)=\varnothing$.

Case B: $\quad p(h)=(w, T)$, and either $\hat{z}_{j}(h) \leq z_{j}(h)$ or for some $i \in T A(h)$ it is the case that $\hat{z}_{i}(h)<z_{i}(h)$.

Case C: $\quad p(h)=(w, T), \hat{z}_{j}(h)>z_{j}$ and for all $i \in T A(h)$ it is the case that $\hat{\mathbf{z}}_{\mathbf{i}}(\mathbf{h}) \geq \mathbf{z}_{\mathbf{i}}(\mathbf{h})$.

Note that $A$ covers all instances in which $p(h)=\varnothing$ (and more) and $B$ and $C$ cover all other cases. Since the argument applying to cases A and B have a common component, we treat these two cases together for a time, then separating them when necessary.

\section{Cases A or B:}

By employing $f_{j}$, player $j$ will obtain a payoff of $z_{j}(h)$. This is straightforward for case B and follows from Lemma 2 for case $A$. It suffices to show that $z_{j}(h) \geq z_{j}\left(h^{\prime} \mid t^{\prime}\right)$. So, suppose by way of contradiction that $\mathrm{z}_{\mathrm{j}}(\mathrm{h})<\mathrm{z}_{\mathbf{j}}\left(\mathrm{h}^{\prime} \mid \mathrm{t}^{\prime}\right)$

Let $t^{*}=\inf \left\{\hat{t} \in\left[t, t^{\prime}\right] \mid z_{j}(h)<z_{j}\left(h^{\prime} \mid t^{\prime}\right)\right\}$. We claim that $z_{j}(h) \geq z_{j}\left(h^{\prime} \mid t^{*}\right)$. For suppose not. Then $z_{j}(h)<z_{j}\left(h^{\prime} \mid t^{*}\right)=z_{j}\left(\Pi\left(h^{\prime} \mid t^{*}\right), N\left(h^{\prime} \mid t^{*}\right)\right)$. Now by (S4), according to $h^{\prime}$, every player is quiet for times sufficiently near $\mathrm{t}^{*}$. But this implies that for some $\varepsilon>0$ and small enough, $\Pi\left(h^{\prime} \mid t^{*}-\varepsilon\right)=\Pi\left(h^{\prime} \mid t^{*}\right)$ and $N\left(h^{\prime} \mid t^{*}-\varepsilon\right)=N\left(h^{\prime} \mid t^{*}\right)$. Hence, $z_{j}\left(h^{\prime} \mid t^{*}-\varepsilon\right)=z_{j}\left(h^{\prime} \mid t^{*}\right)>z_{j}(h)$ 
contradicting the definition of $t^{*}$. We conclude that indeed $z_{j}(h) \geq z_{j}\left(h^{\prime} \mid t^{*}\right)$, as claimed. Note then that $t^{*}<t^{\prime}$.

Therefore, for some sequence of positive numbers $\left\{\varepsilon_{\mathrm{n}}\right\}$ converging to zero, we have $z_{j}\left(h^{\prime} \mid t^{*}+\varepsilon_{n}\right)>z_{j}(h) \geq z_{j}\left(h^{\prime} \mid t^{*}\right)$. But this can happen, again by (S4), only if according to $h^{\prime}$, at time $t^{*}$ either the current proposal is accepted by a coalition containing $j$, or someone leaves the game. The latter is not possible since $j$ leaves at $t^{\prime}>t^{*}$ and according to their strategies if some $i \neq j$ leaves at $t^{*}<t^{\prime}$, so must $j$. Hence, according to $h^{\prime}$, at time $t^{*}$ the current proposal $p\left(h^{\prime} \mid t^{*}\right)=(y, S)$ with $j \in S$, is accepted.

Now, because $\hat{z}_{j}\left(h^{\prime} \mid t^{*}\right)$ is precisely the resulting value of $z_{j}(\cdot)$ were the current offer accepted, and because by (S4) all players are quiet for times near enough $t^{*}$, we have $\hat{z}_{j}\left(h^{\prime} \mid t^{*}\right)=z_{j}\left(h^{\prime} \mid t^{*}+\varepsilon_{n}\right)$ for $n$ large enough. But $z_{j}\left(h^{\prime} \mid t^{*}+\varepsilon_{n}\right)>z_{j}\left(h^{\prime} \mid t^{*}\right)$ then implies that $\hat{\mathbf{z}}_{\mathrm{j}}\left(\mathrm{h}^{\prime} \mid \mathrm{t}^{*}\right)>\mathrm{z}_{\mathrm{j}}\left(\mathrm{h}^{\prime} \mid \mathrm{t}^{*}\right)$. Since by part (c) of Lemma $1, \sum_{\mathrm{i} \in \mathrm{S}} \hat{\mathbf{z}}_{\mathrm{i}}\left(\mathrm{h}^{\prime} \mid \mathrm{t}^{*}\right)=\sum_{\mathrm{i} \in \mathrm{S}^{\mathrm{z}}} \mathrm{z}_{\mathrm{i}}\left(\mathrm{h}^{\prime} \mid \mathrm{t}^{*}\right)$, there must, since $j \in S$, be a $k \in S$ such that $\hat{z}_{k^{\prime}}\left(h^{\prime} \mid t^{*}\right)<z_{k}\left(h^{\prime} \mid t^{*}\right)$. We now continue from this point with case A only. The continuation of the argument for case B will be provided immediately after. Case A: $A(h)=\phi$.

Now, because at time $t^{*}$ the proposal $(y, S)$ is accepted by all members of $S$ who have not yet accepted it, and $A(h)=\phi$, there is a time $t_{k} \in\left[t, t^{*}\right]$ such that player $k$ according to $h^{\prime}$ accepts $(y, S)$ at time $t_{k}$. But since $k \neq j$ is playing according to $f_{k}$, it must then be the case that $\hat{z}_{k}\left(h^{\prime} \mid t_{k}\right) \geq z_{k}\left(h^{\prime} \mid t_{k}\right)$. But since the current proposal, the collection of accepted proposals, and the player remaining in the game are unchanged from $t_{k}$ to $t^{*}$ we have $\hat{z}_{k}\left(h^{\prime} \mid t_{k}\right)=\hat{z}_{k}\left(h^{\prime} \mid t^{*}\right)$ and $z_{k}\left(h^{\prime} \mid t_{k}\right)=z_{k}\left(h^{\prime} \mid t^{*}\right)$. But this contradicts $\hat{z}_{k}\left(h^{\prime} \mid t^{*}\right)<z_{k}\left(h^{\prime} \mid t^{*}\right)$.

We conclude that $z_{j}(h) \geq z_{j}\left(h^{\prime} \mid t^{\prime}\right)$ so that the strategy $f_{j}^{\prime}$ is no better for $j$ than $f_{j}$ given h. This completes the argument for case A. We now pick up where we left off and conclude the argument for case $B$.

Case $B: p(h)=(w, T)$ and for some $i \neq j i \in T A(h)$, it is the case that $\hat{z}_{i}(h)<z_{i}(h)$ or $\hat{z}_{j}(h) \leq z_{j}(h)$. Claim: According to $h^{\prime}$, the proposal $p(h)=(w, T)$ is not accepted by all members of $T$ and is subsequently replaced by the proposal $(y, S)$. 
Proof of Claim: Since $(y, S)$ is proposed at time $t^{*}$ according to $h^{\prime}$, it suffices to show that according to $h^{\prime},(w, T)=p(h)$ is not accepted by all members of $T$. If for some $i \neq j, i \in T A(h)$ and $\hat{z}_{i}(h)<z_{i}(h)$, then according to $f_{i}$ player $i$ will not accept $p(h)$. Since, while the proposal remains $\mathrm{p}(\mathrm{h}), \hat{z}_{\mathbf{i}}(\cdot)$ and $\mathrm{z}_{\mathbf{i}}(\mathrm{h})$ remain constant (recall that the collection of remaining players is constant from $t$ to $t^{\prime}$ according to $h^{\prime}$ ), player $i$ will not, according to $f_{i}$, accept $(w, T)$ so long as it remains the current proposal according to $h$. The only other possibility is that $\hat{z}_{j}(h) \leq z_{j}(h)$ and for $i \neq j$ and $i \in T A(h) \hat{z}_{i}(h) \geq z_{i}(h)$. But in this case, if $j$ accepts $p(h)$, then it will be accepted by all remaining $i \in \operatorname{TA}(h)$ according to $f_{i}$. Subsequently, there will be a period of time during which all players are quiet, the value of $z_{j}(h)$ will be $\hat{z}_{j}(h)$, and during which no player will have accepted the current offer (if there is one). Since by case A it is optimal from this point on for $j$ to play according to $f_{j}$, his resulting payoff (by Lemma 2) will be no greater than $\hat{z}_{j}(h) \leq z_{j}(h)$. But $j^{\prime}$ s resulting payoff from $f_{j}^{\prime}$ is $z_{j}\left(h^{\prime} \mid t^{\prime}\right)$. Hence, $z_{j}\left(h^{\prime} \mid t^{\prime}\right) \leq \hat{z}_{j}(h) \leq z_{j}(h)$. But this contradicts the assumption that $\mathrm{z}_{\mathrm{j}}\left(\mathrm{h}^{\prime} \mid \mathrm{t}^{\prime}\right)>\mathrm{z}_{\mathrm{j}}(\mathrm{h})$, and completes the claim.

By the claim, the proposal $(y, S)$ is made at time t or later according to $h^{\prime}$. In addition, $(y, S)$ is accepted by every member of $S$ on or before time $t^{*}$. Hence, there is a time $t_{k} \in\left[t, t^{*}\right]$ such that player $k$ (that player satisfying $\hat{z}_{k}\left(h^{\prime} \mid t^{*}\right)<z_{k}\left(h \mid t^{*}\right)$ from the argument above applying to both cases $A$ and $B$ ), according to $h^{\prime}$ accepts $(y, S)$ at time $t_{k}$. The conclusion reached in the last two paragraphs of the argument in case $A$ now goes through as before. This completes the argument for case B.

Case $C: p(h)=(w, T), \hat{z}_{j}(h)>z_{j}(h)$, and for every $i \in T A(h), \hat{z}_{i}(h) \geq z_{i}(h)$.

If all players play according to $f_{i}$, then $j$ obtains the payoff $\hat{z}_{j}(h)$, since $j \notin A(h)$. We wish to show that the strategy $f_{j}^{\prime}$ does not yield $j$ a higher payoff, i.e. that $\hat{z}_{j}\left(h^{\prime} \mid t^{\prime}\right)<\hat{z}_{j}(h)$.

Now, all $i \in T A(h)$ other than $j$ (note that $\hat{z}_{j}(h)>z_{j}(h)$ implies $j \in T$ ) will, according to $f_{i}$, accept the proposal $(w, T)$ if it is not replaced by a new proposal. Also, all if $T$ will, according to $f_{i}$, be quiet until $(w, T)$ is accepted or replaced by a new proposal. So, if $j$ accepts $(w, T)$ before making a new proposal, then $(w, T)$ will be accepted by all members of $T$. 
Subsequently, there will (for a period of time) be no current offer. By case A, it is then optimal for $j$ to employ $f_{j}$ and thereby obtain $\hat{z}_{j}(h)$, the new value of $z_{j}$. Thus $j$ cannot improve upon $f_{j}$ by accepting $(w, T)$ before making a new proposal.

So, suppose that before $\mathrm{j}$ accepts $(w, T), j$ makes a new proposal, $\left(w^{\prime}, T^{\prime}\right)$. For a period of time subsequent to $j$ 's proposal the current proposal will be $\left(w^{\prime}, T^{\prime}\right)$, no player will have accepted it, and the value of $z_{j}$ will remain equal to $z_{j}(h)$. Since, during this period, no player has accepted the current offer, the analysis of case $A$ indicates that $j$ can do no better than obtain a payoff of $z_{j}=z_{j}(h)$. Since $z_{j}(h)<\hat{z}_{j}(h)$ we conclude that $j$ cannot improve upon $f_{j}$ by making a proposal and not accepting $(w, T)$.

The only remaining option is for $j$ to leave before making a proposal and before accepting $(w, T)$. But this yields $j$ a payoff no greater than $z_{j}(h)$ regardless of when $j$ leaves since the others, according to their strategies, either are quiet or accept the current offer. But $z_{j}(h) \leq \hat{z}_{j}(h)$ and so this is no better than employing $f_{j}$ either. This completes the argument for case $\mathrm{C}$.

We say that an allocation $x$ belongs to the $\varepsilon$-core of the game $(v, N)$ if for every coalition $S \subseteq N$, whenever $\sum_{i \in S} y_{i} \leq v(S)$, it is the case that $y_{j} \leq x_{j}+\varepsilon$ for some $j \in S$. We also define a profile of stationary strategies $\mathrm{f}^{*}$ to be an $\varepsilon$-SSPE if for any history $\mathrm{h} \in \mathrm{H}$ and for all $\mathrm{i}$, $u_{i}\left(f^{*} \mid h\right)+\varepsilon \geq u_{i}\left(\left(f^{*}{ }_{-i}, f_{i}\right) \mid h\right)$, for all $f_{i} \in F_{i}$

Theorem 3: If payoffs are exponentially discounted and $G=(v, N)$ is totally balanced, then $x$ can be supported as an $\varepsilon$-SSPE if and only if $x$ is in the $\varepsilon$-core of $(v, N)$.

Proof: To see that only $\varepsilon$-core allocations can be supported as $\varepsilon$-SSPE strategies one need only apply once again the proof of Theorem 1 with the obvious changes. To see that any $\varepsilon$-core allocation can be supported as an $\varepsilon$-SSPE it suffices to employ the strategies provided in the proof of Theorem 2 with the following modifications. Let $T_{\Delta}=\{\Delta, 2 \Delta, 3 \Delta, \ldots\}$ for $\Delta>0$. 
Replace in (i) the phrase "if $\tau(h)$ is not a positive integer" by "if $\tau(h) \notin T_{\Delta}$, and replace in (ii) the phrase "if $\tau(\mathrm{h})$ is a positive integer" by "if $\tau(\mathrm{h}) \in \mathrm{T} \Delta^{.}$" For $\Delta$ close enough to zero, the resulting strategies will constitute an $\varepsilon$-SSPE.

Q.E.D.

Remark 4: The result expressed in Theorem 3 should be contrasted with what one would expect were discounting introduced in a discrete time setting. Together with stationarity, discounting and discrete time would, by virtue of Rubinstein-like arguments, not allow one to support every core allocation as an SSPE. And this would be so for all $\varepsilon>0$ small enough. The presence of continuous time is critical in order that the results be robust to the inclusion of time-preference.

Remark 5: Exponential discounting is not essential. Any manner of discounting that is continuous in time is also covered by the same proof. 


\section{Appendix}

Lemma 1: For every $h \in H$, if $p(h)=(y, S)$ and $\Pi(h)=\left\{\left(y^{1}, S^{1}\right), \ldots,\left(y^{m}, S^{m}\right)\right\}$, then

(a) $z_{i}(h) \geq y_{i}^{k}$ for every $k=1, \ldots, m$ and $i \in S^{k}$,

(b) $\sum_{i \in S} \mathrm{k}^{\mathrm{z}_{i}}(\mathrm{~h})=\sum_{\mathrm{i} \in \mathrm{S}^{\mathrm{k}}} \mathrm{x}_{\mathrm{i}}(\mathrm{N}(\mathrm{h}))$ for every $\mathrm{k}=1, \ldots, \mathrm{m}$ and

(c) $\sum_{i \in S} z_{i}(h)=\sum_{i \in S} \hat{z}_{i}(h)$.

Proof: (a) For every $k, \sum_{i \in S^{k}} y_{i}^{k} \leq v\left(S^{k}\right)$ since $\left(y, S^{k}\right)$ is a proposal. In addition, because $x(N(h))$ is in the core of $(v, N(h))$ and $S^{k} \subseteq N(h)$ we have $\sum_{i \in S^{k}} x_{i}(N(h)) \geq v\left(S^{k}\right)$. Hence $\sum_{i \in S} k^{k}\left(x_{i}(N(h))-y_{i}^{k}\right) \geq 0$. This, together with the definition of $z_{i}(h)$ yields (a).

(b) This follows trivially from the definition of $z_{i}(h)$.

(c) Applying (b) to the RHS and using the definition of $\hat{z}_{\mathbf{i}}$ (h) yields $\sum_{i \in S} \hat{z}_{i}(h)=\sum_{i \in S} x_{i}(N(h))$. The LHS has the same sum since it is precisely (b) summed over $\mathrm{k}=1, \ldots, \mathrm{m}$.

Q.E.D.

Lemma 2: For any $t \geq 0$ and $h \in H(t)$, if $A(h)=\phi$, then the outcome generated by $\left(f_{1}, \ldots, f_{n}\right)$ after $h$ is $z(h)$.

Proof: Let $\overline{\mathrm{t}}$ be the smallest time at least as large as $\mathrm{t}$ such that $\overline{\mathrm{t}}-\tau(\mathrm{h})$ is an integer. According to $f$, all players are quiet between $t$ and $\bar{t}$. Call the history so far generated $\bar{h} \in H(\bar{t})$. Now if according to $\bar{h}, f_{i}(\bar{h})=\ell$, then the outcome will clearly be $z(\bar{h})$ and this is equal to $z(h)$. If $f_{i}(\bar{h})=(z(\bar{h}), N(\bar{h}))$, then between $\bar{t}$ and $\overline{\bar{t}}=\bar{t}+1$, all players will be quiet according to $f$. Call the history generated this way $\overline{\bar{h}} \in H(\overline{\bar{t}})$. Since $p(\overline{\bar{h}})=(z(\bar{h}), N(\bar{h}))$, we have $\hat{z}_{i}(\overline{\bar{h}})=z_{i}(\bar{h})$. To see this, note that since no player has left between $t$ and $\overline{\bar{t}}, N(h)=N(\bar{h})=N(\overline{\bar{h}})$ and by definition: 
$\hat{z}_{i}(\bar{h})=z_{i}(\bar{h})+\frac{\sum_{j \in N\left(h^{\prime}\right)}\left(x_{j}(N(\bar{h}))-z_{i}(\bar{h})\right)}{|N(h)|} \quad$ for all $i \in N(\bar{h})$.

But now use part (b) of Lemma 1 to conclude that $\hat{z}(\bar{h})=z_{i}(\bar{h})$. Since no offer has been accepted between $t$ and $\overline{\bar{t}}$ according to $\overline{\bar{h}}$, we have $z_{i}(\overline{\bar{h}})=z_{i}(\bar{h})=z_{i}(h)$ for every $i \in N(h)$. Hence $\hat{z}_{i}(\overline{\bar{h}})=z_{i}(\overline{\bar{h}})$. Hence, given $\overline{\bar{h}}$, according to $f$, all players in $N(h)=N(\bar{h})$ accept $p(\overline{\bar{h}})=(z(h), N(h))$. Finally, at time $\overline{\bar{t}}=\overline{\bar{t}}+1$ all players, having been quiet between $t$ and $\overline{\overline{\mathrm{t}}}$ leave according to $\mathrm{f}$, yielding the outcome $\mathrm{z}(\mathrm{h})$.

Finally, suppose that $p(\bar{h})=(y, S)$ and that $f_{i}(\bar{h})=a$ for some $i \in S$. Since $A(h)=A(\bar{h})=\phi$, this means that $\hat{z}_{i}(\bar{h}) \geq z_{i}(\bar{h})$ for every $i \in S$, and $f_{i}(\bar{h})=q$ for all other $i$. Moreover, by part $(c)$ of Lemma 1 it must then be the case that $\hat{z}_{i}(\bar{h})=z_{i}(\bar{h})$ for all $i \in S$. Since for all $i \in N(\bar{h}) \backslash S$, $\hat{z}_{i}(\bar{h})=z_{i}(\bar{h})$, we then have $\hat{z}_{i}(\bar{h})=z_{i}(\bar{h})$ for all $i \in N(\bar{h})$. According to $f$, all players are quiet until time $\overline{\bar{t}}=\bar{t}+1$. Let $\overline{\mathrm{h}} \in \mathrm{H}(\overline{\overline{\mathrm{t}}})$ be the history so far generated. Accordingly, $\mathrm{p}(\overline{\mathrm{h}})=\phi$ so that $f_{i}(\overline{\bar{h}})=(z(\overline{\bar{h}}), N(\overline{\bar{h}}))$. But for every $i \in N(\overline{\bar{h}})=N(h), \hat{z}_{i}(\overline{\bar{h}})=\hat{z}_{i}(\bar{h})=z(h)$, so that $f_{i}(\overline{\bar{h}})=(z(h), N(h))$. As before, in one unit of time all players accept $(z(h), N(h))$ and in another unit of time all players leave.

Q.E.D. 


\section{References}

Bergin, J. and B. MacLeod (1989): "Renegotiation Proof Equilibria in Continuous Time Games," Discussion Paper \#753, Department of Economics, Queens University.

Binmore, K. G. (1985): "Bargaining and Coalitions," in Roth, Alvin E. (Ed.), Game Theoretic Model of Bargaining (Cambridge University Press).

Chatterjee, K., B. Dutta, D. Ray, and K. Sengupta (1990): "A Noncooperative Theory of Coalition Bargaining," Working paper.

Edgeworth, F.Y. (1881): "Mathematical Psychics," Reprinted 1967 by Augustus M. Kelley Publishers.

Gale, D. (1987): "Limit Theorems for Market with Sequential Bargaining," JET, 43, 20-54.

Gul, F. (1989): "Bargaining Foundation of Shapley Value," Econometrica, 57, 81-95.

Harsanyi, J. C. (1974): "An Equilibrium-Point Interpretation of Stable Sets and a Proposed Alternative Definition," Management Science, 20, 1422-1495.

Kalai, E., E. Postlewaite, and J. Roberts (1979): "A Group Incentive Compatible Mechanism Yielding Core Allocations," JET, 20, 13-22.

Moldovanu, B. and E. Winter (1991): "Order Independent Equilibria," Working Paper.

Perry, M. and P. Reny (1991): "A Non-Cooperative Bargaining Model with Strategically Timed Offers," mimeo, Department of Economics, The University of Western Ontario.

Rubinstein, A. and A. Wolinsky (1985): "Equilibrium in a Market with Sequential Bargaining," Econometrica 52, 1333-1150.

Selten, R. (1981): "A Noncooperative Model of Characteristic Function Bargaining," in Bohm, . and H. H. Nachtkamp (Ed.), Essays in Game Theory and Mathematical Economics in Honor of Oskar Morgenstern, 131-151 (Bibliographisches Institut Mannheim).

Stinchcombe, M. (1988): "Maximal Strategy Sets for Continuous-Time Game Theory", Discussion Paper, Department of Economics, , U.C. Berkley. 